\title{
Comparison of Glutues Maximus Activation to Flexion Bias Exercises Along with Met Technique in Subjects with Anterior Rotated Sacroiliac Joint Dysfunction-a Randomised Control Trial
}

\author{
Vaidya Sanika, PT, Venkatesan Prem, PT, PHD, ${ }^{*}$ Harikesan Karvannan, PT, PHD*
}

Department of Physiotherapy, Manipal University, School of Allied Health Sciences, Bangalore, India

Background: Sacroiliac joint dysfunction (SIJD) is the primary source of lowback pain. Main muscles forming the force closure of sacroiliac joint are the biceps femoris and gluteus maximus which increase the stability through massive attachments via sacrotuberous ligament. However, there is a dearth of literature of the importance of activation of gluteus maximus in SIJD.

Purpose: To study the effect of gluteus maximus activation on Oswestry Disability Index (ODI), visual analog scale (VAS), and pelvic tilt angle in subjects with anterior rotated sacrolilac joint dysfunction.

Settings: The study was conducted in outpatient Physiotherapy Department, Manipal Hospital, Bangalore, India.

Participants: Anterior rotated SIJD subjects were recruited in the study. They were divided into two groups (experimental and control groups) by block randomisation.

Research Design: This is a randomised control trial.

Treatment: Treatment order was determined by block randomisation. The subjects of both experimental and control group received MET technique on $1^{\text {st }}$ session to correct the anterior rotated SIJD. The experimental group received gluteus maximus activation protocol, whereas the control group received flexion bias exercises. The groups received the treatment of 20 mins per session. There were two supervised sessions per week for four weeks.

Main Outcome Measures: The primary outcome measure in the study is Oswestry Disability Index (ODI). The secondary outcome measures included visual analog scale (VAS) and Palpation Meter (PALM).

Results: 48 subjects (26 females, 22 males) were randomised into experimental and control groups having anterior rotation SIJD, and average age in groups was $38.83 \pm 11.4$ years and $34.96 \pm 9.5$ years, respectively. The within-group analysis showed significant improvements in only ODI outcome of both the groups ( $p=$ .001). The between-group analysis in both groups did not show any statistical significant difference in ODI, VAS, or PALM.

Conclusion: The flexion bias exercise and the gluteus maximus activation exercises used in this study were equally effective in improving physical function and reduction in pain, and maintaining the normal pelvic angle in subjects with anterior rotated SIJD.

KEYWORDS: sacroiliac joint dysfunction; gluteus maximus; PALM device; visual analogue scale

\section{INTRODUCTION}

Sacroiliac joint (SIJ) is a primary source of low-back pain. The joint has a complex anatomy and it is ten times more vulnerable than lumbar segments. Axial compression forces and axial torsion overloading lead to sacroiliac joint dysfunction. (1) The prevalence of sacroiliac joint dysfunction is $10 \%-25 \%$ in patients with chronic lowback pain. SIJ dysfunction is common in individuals who participate in activities such as lifting and twisting, and also racket sports where repetitive unilateral loads and unidirectional pelvic shear and/or torsional forces fall, leading to pelvic asymmetry. (2)

SIJ stability is enhanced by four factors viz. form closure, force closure, motor control, emotions and awareness. Form closure and force closure are the selflocking mechanisms of the pelvis, which prevents SIJ pathology. ${ }^{(3)}$ Force closure is important for the stability of the SIJ. The muscles, ligaments, and thoracolumbar fascia help in stabilizing the pelvis in unilateral loading, where the shear forces act on the pelvis. The muscle-ligament-fascia 
system is required to compress the $\mathrm{SIJ}$, thus stabilizing the pelvis. ${ }^{(4-5)}$ The main muscles are biceps femoris, gluteus maximus, and erector spinae which are responsible for stability of SIJ. The attachment of latissimus dorsi and gluteus maximus via sacrotuberous ligament and the thoracolumbar fascia gives required stability to the sacroiliac joint. The load transfer from spine to legs is effectively carried out by contraction of these muscles, increasing the compression of SIJ, thus preventing shear forces. ${ }^{6-7)}$

There are five types of sacroiliac joint dysfunction: anterior rotated sacroiliac joint dysfunction, posteriorly rotated sacroiliac joint dysfunction, up slip, down slip, and sacral torsions. Many of the day-to-day activities involve twisting and lifting of the trunk and pelvis, which leads to the commonly experienced anteriorly rotated sacroiliac joint dysfunction. It causes pain around the posterior superior iliac spine (PSIS) and lengthening of the affected limb leading to an inability to hold the pelvis in a neutral position and unequal weight transfer during walking. There is inhibition of gluteus maximus and inability to provide stability to the pelvis. ${ }^{(8)}$ In sacroiliac joint dysfunction, there is altered recruitment of gluteus maximus during load transfer through the pelvis. Proper activation of gluteus maximus muscle plays a crucial role in stabilisation of the pelvis. ${ }^{(5,8)}$

Gluteus maximus functions primarily as a hip extensor, and also plays an important role in pelvic and spinal stabilisation during load transfer via the thoracolumbar fascia and sacrotuberous ligament. (8) The electromyography study on patients with sacroiliac joint dysfunction concluded that there is delayed activation of gluteus maximus and compensatory hyperactivity of biceps femoris to maintain the stability. ${ }^{(1)}$

The anteriorly rotated sacroiliac joint dysfunction is commonly treated conservatively by correction of dysfunction by muscle energy technique (MET), joint mobilization, electrotherapy modalities, and flexion bias exercises. (9-10) Studies have investigated the use of MET and joint mobilization, along with electrotherapy modalities (ultrasound and TENS), which showed significant improvement in VAS scores and functional status post-treatment of sacroiliac joint dysfunction. The corrective exercises for SIJD include flexion bias exercises as they help to restore the selfbracing position of the joint.(10-11)

There is evidence that both manipulation and stabilisation exercises can reduce pain and disability in patients with sacroiliac joint dysfunction. (12) Furthermore, a case series on SI joint dysfunction has shown improvement in strength, function, and reduction in pain after completing the gluteus maximus strengthening programme. ${ }^{(13)}$

To our knowledge, studies on effectiveness of gluteus maximus activation exercises, along with MET, are not found in the reviewed literature. ${ }^{(14,15)}$ In addition, no studies have compared the effects of gluteus maximus activation exercises to flexion bias exercises on functional disability, pain intensity, and pelvic tilt angles on subjects with anteriorly rotated sacroiliac joint dysfunction.

Hence, the aim of the current study is to compare the effect of gluteus maximus activation exercise to flexion bias exercises, along with MET, on function using Oswestery Disability index (ODI), on pain using the visual analogue scale (VAS), and on pelvic tilt angle using Palpation Meter (PALM) on anteriorly rotated sacroiliac joint dysfunction.

\section{METHODS}

\section{Participants}

The study was approved by Institutional Review Board of School of Allied Health Sciences, Manipal College of Health Professions. Subjects diagnosed with anteriorly rotated sacroiliac joint dysfunction were screened for inclusion in outpatient Physiotherapy Department of Manipal Hospital, Bangalore, India. The therapist screened and recruited the subjects in the study. Written informed consent was obtained from the eligible subjects who met the inclusion criteria. Subjects with anteriorly rotated sacroiliac joint dysfunction were included who met the following criteria: (a) history of unilateral pelvic pain / SI region and not radiating to L5 vertebrae and above; (b) positive findings of dynamic form closure tests such as standing flexion test, sitting flexion test, and stork test; (c) positive active straight leg raise (SLR) test; (d) positive findings of at least three out of five SIJ pain provocation tests. Subjects diagnosed with hip pain, ankylosing spondylosis, lumbar radiculopathy, spinal 
pathologies like spondylosis, spondylolysis and spondylolisthesis, and subjects immediate post-pregnancy were also excluded from the study. (11)

The sample size was calculated using ODI as the primary outcome measure, with $5 \%$ level of significance, $80 \%$ power, anticipating a minimally clinically significant difference of 11.5 and standard deviation (SD) as 14. The dropout rate of 10\% and confidence interval $(\mathrm{Cl})$ of 95\% were set. Sample size estimation revealed 48 subjects ( 24 subjects in each group).

\section{Assessment}

Studies have proved concurrent criterion validity of comprehensive examination which consists of McKenzie Method evaluation in combination with SIJ pain provocation tests. ${ }^{(17)}$

Repeated movement examination was performed according to McKenzie Method evaluation, and subjects with discogenic complaints were excluded from the study.

\section{Outcome Measures}

\section{Oswestry disability index}

Oswestry Disability Index is a questionnaire used to assess disability in chronic low-back pain and sacroiliac joint pain patients by determining its impact on daily activities due to pain. This questionnaire consists of 10 sections describing the pain and its impact. Each section scores from 0 to 5 , 0 being no pain and 5 being severe pain. The domains include pain intensity, personal care, lifting, sitting, walking, standing, sleeping, travel, social life, and change in the degree of pain. The minimal clinically important change score (MCID) ranges from 3.5 to 19.5 for ODI scale, and its minimal detectable change (MDC) is 15.5 . The level of disability is calculated as a percentage, which states 0\%-20\% as minimal disability, $21 \%-40 \%$ as moderate disability, $41 \%-60 \%$ as severe disability, $61 \%-80 \%$ as crippled, and $81 \%-100 \%$ as bed-bound. ${ }^{16)}$ MCID for ODI to be 3.5 to 19.5 points which falls within the previously reported range for lumbar pain and indicates the improvement in disability by $15 \%$ to be beyond variation. ${ }^{(17)}$

\section{Palpation meter (PALM)}

PALM is a device used to measure the pelvic tilt angle of the pelvis. It is used to measure the anterior-posterior pelvic tilt and lateral pelvic tilt angles. ${ }^{(18)}$

The anterior inferior iliac spine (ASIS) and posterior superior iliac spine (PSIS) were palpated to measure the anterior-posterior pelvic tilt, and then the most prominent part of ASIS was marked with markers. The PSIS was then palpated and was marked by tracing the iliac crest posteriorly and then moving thumbs superiorly and laterally from the edge of the sacrum. Then the calipers were placed on the respective markings and the subject was instructed to take a deep breath and hold the breath till the measurement was taken. To measure lateral pelvic tilt the therapist stood behind the subject. The highest point of the iliac crest was marked. The PALM calipers were placed on the marked points and the subject was made to take a deep breath and hold it. To avoid errors, a minimum of two measurements were taken for both AP and lateral pelvic tilts. Out of these two readings, the last reading was taken for analysis. In normal asymptomatic individuals, the pelvic tilt angle is found to be $2^{\circ}-3^{\circ}$ in sagittal plane and $0.8^{\circ}$ in frontal plane. PALM was concluded to be a reliable (inter-rater reliability ICC 0.88 and intra-rater reliability ICC 0.90) and valid (ICC 0.92) instrument by researchers for measuring pelvic crest height difference. Its reliability to measure a pelvic angle is also good.(19)

\section{Visual analog scale}

A $10 \mathrm{~cm}$ visual analog scale (VAS) was used to assess the perceived pain level of the subject. Subjects were asked to rate their pain with zero $(0 \mathrm{~cm})$ being no pain and ten $(10 \mathrm{~cm})$ being the worst pain they could imagine. The inter-rater reliability of VAS is good, with an inter-class coefficient (ICC) of 0.97. Two cm difference on VAS represents a smallest measurable change in pain intensity, which is clinically important in chronic musculoskeletal pain. (20)

\section{Procedure}

Baseline data for ODI, Palpation meter (PALM), and VAS were recorded by the therapist before MET correction of the subjects in both the groups.

Anterior rotated sacroiliac joint dysfunction subjects were recruited in the study, were divided into two groups (experimental and control group) by block randomization. A total of eight blocks were made, each block consisted of six subjects (three 
control and three experimental), resulting in a total sample size of 24 in each group. The experimental group received MET and gluteus maximus activation exercise protocol. The control group received MET and flexion bias exercise protocol. The data for VAS and PALM were collected by the same therapist immediately after correction of pelvic tilt angle by MET technique. Post four weeks of exercise sessions, outcome data (ODI, PALM, and VAS) of subjects in both experimental and control groups was recorded by the therapist. The outcome assessor was not blinded. A written exercise protocol was provided for home programme. There was no risk of harm of any exercises reported by any subject in the study.

\section{Intervention}

Muscle energy technique was used by the therapist to correct anteriorly rotated sacroiliac joint dysfunction. The subject was made to lie in supine position and perform a bridge to square the pelvis. The subject then straightened the legs and therapist checked the level of both the medial malleoli with his thumbs placed below the malleoli. If the leg was found to be lengthened on the involved side, anteriorly rotated sacroiliac joint dysfunction was confirmed. The therapist held the involved leg in hip and knee flexion around $40^{\circ}$ to $50^{\circ}$, and placed the leg under axilla and performed a controlled isometric contraction of the hip extensors (only $20 \%$ of maximum contraction) for about 5-10 sec and repeated the procedure twice. The pelvis was squared again and the leg length was observed by comparing the two malleoli. This technique is called the 'self-bracing' position for sacroiliac joint dysfunction. It moves the PSIS posteriorly, caudally, and medially on the sacrum where fixation occurs. ${ }^{(15)}$

The control group received standard rehabilitation protocol that focused on correction of anteriorly rotated sacroiliac joint dysfunction by MET. Immediately after the correction, PALM and VAS outcome measures were taken. Flexion bias exercises were taught which included knee to chest, crunches, cat and camel, and long sitting flexion exercises. Each session lasted for 20 min, twice a week for four continuous weeks. All exercises were started with 10-sec hold, 10 repetitions and 1 set, repeated two times a day. The sessions were supervised for two days a week. The subjects were asked to continue the exercises at home. A home programme of five days was unsupervised. (9-10)

The experimental group was given correction of anteriorly rotated sacroiliac joint dysfunction by MET following which immediately PALM and VAS outcome measures were taken. Gluteus maximus activation exercises were taught, which included activation in crook lying, bridging, prone lying hip lift, and sitting waiter's bow. Each session lasted for 20 min twice a week for four continuous weeks. All exercises were started with 10-sec hold, 10 repetitions and 1 set, repeated two times a day. The sessions were supervised for two days in a week. The subjects were asked to continue the exercises at home. A home programme of five days was unsupervised.(15)

\section{Statistical Analyses}

The statistical analyses were performed using IBM SPSS Statistics, version 20.0 (SPSS Inc., Armonk, NY). Baseline normality was checked using Q-Q plot test for age, gender, chronicity, ODI, PALM, and VAS. A paired $t$ test was used for ODI outcome measure for within-group analysis from baseline to post four weeks of intervention. The Independent $t$ test was used for ODI outcome measure for between-group analysis. Repeated measure ANOVA test was used for PALM and VAS outcome measures, for within-group analysis, and between-group analysis. Outcomes were taken from baseline to immediate correction of the dysfunction by MET and post four weeks of intervention. A $p$ value of $<.05$ was considered statistically significant.

\section{RESULTS}

Eighty subjects were screened for the study. Forty-eight subjects were enrolled in the study according to eligibility criteria. Baseline outcome measures were taken pre-treatment. All subjects were randomly allocated to experimental $(n=24)$ and control group $(n=24)$. The experimental group received MET correction and gluteus maximus activation exercises. The control group received MET correction and flexion bias exercises. The data were normally distributed, and the results are projected as mean and standard deviation of PALM (in degrees) and VAS scores (in $\mathrm{cm}$ ). The 
results of the ODI are projected in mean percentage. The mean age of subjects in the experimental group is $38.83 \pm 11.4$ (in years) and in the control group is $34.96 \pm 9.5$ (in years) (Table 1). See flowchart (Figure 1) for details of randomisation. There were no adverse effects experienced by any of the subjects throughout the study.

The within-group analysis in the experimental group and control group showed improvement in ODI scores from 35\% to $7.25 \%$ and $37.7 \%$ to $14.5 \%$, respectively, post four weeks of intervention, and demonstrated $p$ value of .001 which is statistically significant (Tables 2 and 3). In the betweengroup analysis, the disability index in experimental group was $7.25 \%$, whereas it was $14.5 \%$ in control group in ODI outcome measured post four weeks of intervention. The result demonstrated $p$ value of .011 which is statistically significant. The MCID for within and between group analyses for ODI is $15.5 \%{ }^{(17)}$ (Table 4).

The immediate effect of MET on VAS scores showed reduction from $6.79 \mathrm{~cm}$ to $4.33 \mathrm{~cm}$ in experimental group and $6.79 \mathrm{~cm}$ to $4.08 \mathrm{~cm}$ in control group, respectively. The scores of VAS demonstrated $p$ value of .112 and .658 , respectively, which are statistically insignificant. The within-group analysis of VAS scores post four weeks showed reduction in pain from $4.33 \mathrm{~cm}$ to $1.17 \mathrm{~cm}$ in experimental group and $4.08 \mathrm{~cm}$ to $1.50 \mathrm{~cm}$ in control group, respectively. The $p$ value

TABLE 1. Demographics and Baseline Outcome Measures $^{a}$

\begin{tabular}{lccc}
\hline \multicolumn{1}{c}{ Variables } & $\begin{array}{c}\text { Experimental } \\
(n=24) \\
\text { Mean } \pm S D\end{array}$ & $\begin{array}{c}\text { Control }(n=24) \\
\text { Mean } \pm S D\end{array}$ & $\begin{array}{c}p \\
\text { value }\end{array}$ \\
\hline $\begin{array}{l}\text { Age (in years) } \\
\text { Gender }\end{array}$ & $38.83 \pm(11.4)$ & $34.96 \pm(9.5)$ & .209 \\
$\begin{array}{l}\text { Female: Male } \\
\begin{array}{l}\text { Chronicity } \\
\text { (in months) }\end{array}\end{array}$ & 2.1 & $2: 1$ & $.568 \pm(9.613)$ \\
$\begin{array}{l}\text { Pre-ODI (in \%) } \\
\begin{array}{l}\text { Pre-PALM } \\
\text { (in degrees) }\end{array}\end{array}$ & $35 \pm(8)$ & $37.7 \pm(7.32)$ & .227 \\
$\begin{array}{l}\text { Pre-VAS } \\
\text { (in cm) }\end{array}$ & $6.79 \pm(1.587)$ & $6.79 \pm(1.31)$ & 1.000 \\
\hline
\end{tabular}

aBaseline normality was checked using Q-Q plot test for age, gender, chronicity, ODI, PALM, and VAS. ODI = Oswestery Disability Index; VAS = visual analog scale; PALM = Palpation Meter.

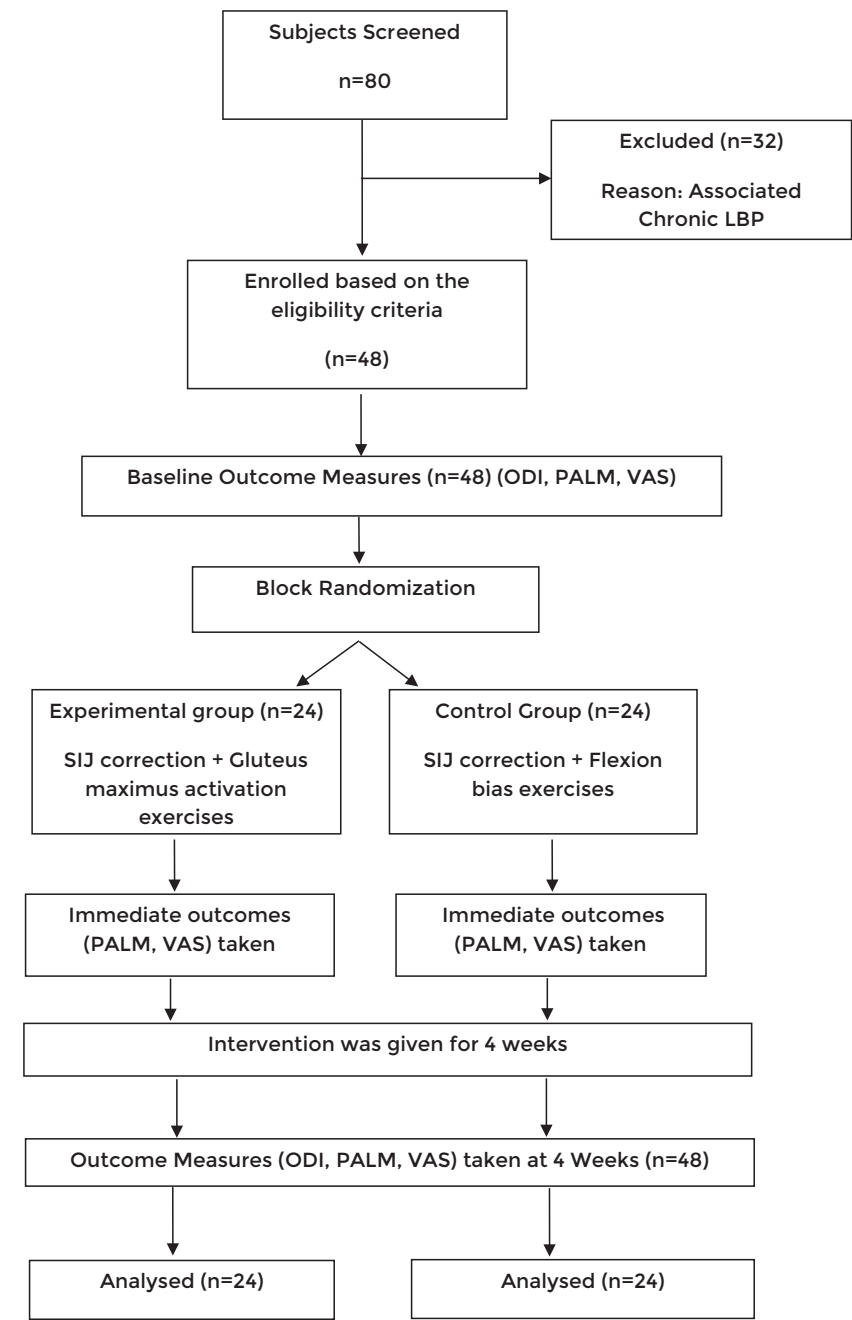

FIGURE 7. Consort flowchart of the study.

in both the groups was not statistically significant. MCID for VAS for within-group and between-group analysis is $2 \mathrm{~cm}$. The between group analysis reported change in VAS scores in experimental group as $4.33 \mathrm{~cm}$ compared to control group at 4.08 $\mathrm{cm}$ immediately after MET correction. The change in VAS scores post four weeks of intervention demonstrated as $1.17 \mathrm{~cm}$ in experimental group and $1.50 \mathrm{~cm}$ in control group. The result demonstrated $p$ value of .384 which is not statistically significant.

The immediate effect of MET demonstrate significant change in pelvic tilt angles in experimental group $\left(4.25^{\circ}-2.00^{\circ}\right)$ as well as in control group $\left(4.33^{\circ}-2.00^{\circ}\right)$.

At the end of four weeks, PALM scores were maintained to $2^{\circ}$ in both experimental and control group. MCID for PALM is $2^{\circ}-3^{\circ} .{ }^{18)}$ The between-group analysis in both the groups did not show statistically significant difference. 
TABLE 2. Within-Group Analysis of Experimental Group

\begin{tabular}{|c|c|c|c|c|}
\hline & \multicolumn{3}{|c|}{ Experimental Group } & \multirow[b]{2}{*}{ p value } \\
\hline & $\begin{array}{c}\text { Pre (Baseline) } \\
\text { Mean } \pm S D\end{array}$ & $\begin{array}{l}\text { Immediate } \\
\text { Mean } \pm S D\end{array}$ & $\begin{array}{c}\text { Post } 4 \text { Weeks } \\
\text { Mean } \pm S D\end{array}$ & \\
\hline ODIa (in \%) & $35 \pm(8)$ & --- & $7.25 \pm(2.690)$ & $<.001$ \\
\hline PALM ${ }^{b}$ (in degrees) & $4.25 \pm(0.608)$ & $2.00 \pm(0.00)$ & $2.00 \pm(0.00)$ & --- \\
\hline VAS $^{b}$ (in cm) & $6.79 \pm(1.587)$ & $4.33 \pm(1.274)$ & $1.17 \pm(1.239)$ & .112 \\
\hline
\end{tabular}

aP values are reported using paired $t$ test for ODI.

bRM-ANOVA test used for VAS and PALM.

ODI = Oswestery Disability Index; VAS = visual analog scale; PALM = Palpation Meter.

TABLE 3. Within-Group Analysis of Control Group

\begin{tabular}{|c|c|c|c|c|}
\hline & \multicolumn{3}{|c|}{ Control Group } & \multirow[b]{2}{*}{$p$ value } \\
\hline & $\begin{array}{c}\text { Pre (Baseline) } \\
\text { Mean } \pm S D\end{array}$ & $\begin{array}{l}\text { Immediate } \\
\text { Mean } \pm S D\end{array}$ & $\begin{array}{c}\text { Post } 4 \text { Weeks } \\
\text { Mean } \pm S D\end{array}$ & \\
\hline ODIa (in \%) & $37.7 \pm(7.321)$ & --- & $14.5 \pm(4.943)$ & $<.001$ \\
\hline PALM ${ }^{b}$ (in degrees) & $4.33 \pm(0.565)$ & $2.00 \pm(0.00)$ & $2.00 \pm(0.00)$ & --- \\
\hline VAS $^{b}$ (in cm) & $6.79 \pm(1.318)$ & $4.08 \pm(1.412)$ & $1.50 \pm(1.383)$ & .658 \\
\hline
\end{tabular}

aP values are reported using paired $t$ test for ODI.

${ }^{b}$ RM-ANOVA test used for VAS and PALM.

ODI = Oswestery Disability Index; VAS = visual analog scale; PALM = Palpation Meter.

TABLE 4. Between-Group Analysis

\begin{tabular}{|c|c|c|c|}
\hline & $\begin{array}{c}\text { Experimental } \\
\text { Mean } \pm S D\end{array}$ & $\begin{array}{c}\text { Control } \\
\text { Mean } \pm S D\end{array}$ & $\begin{array}{c}p \\
\text { value }\end{array}$ \\
\hline Pre-ODI (in \%) & $35 \pm(8)$ & $37.7 \pm(7.321)$ & .670 \\
\hline $\begin{array}{l}\text { Post } 4 \text { Weeks } \\
\text { ODla (in \%) }\end{array}$ & $7.25 \pm(2.690)$ & $14.5 \pm(4.943)$ & .011 \\
\hline $\begin{array}{l}\text { Pre-PALM } \\
\text { (in degrees) }\end{array}$ & $4.25 \pm(0.608)$ & $4.33 \pm(0.565)$ & .625 \\
\hline $\begin{array}{l}\text { Immediate } \\
\text { PALM }{ }^{b} \text { (in } \\
\text { degrees) }\end{array}$ & $2.00 \pm(0.00)$ & $2.00 \pm(0.00)$ & --- \\
\hline $\begin{array}{l}\text { Post } 4 \text { Weeks } \\
\text { PALM }{ }^{\mathrm{b}} \text { (in } \\
\text { degrees) }\end{array}$ & $2.00 \pm(0.00)$ & $2.00 \pm(0.00)$ & --- \\
\hline Pre-VAS ${ }^{b}$ (in cm) & $6.79 \pm(1.587)$ & $6.79 \pm(1.318)$ & 1.000 \\
\hline $\begin{array}{l}\text { Immediate VAS } \\
\text { (in cm) }\end{array}$ & $4.33 \pm(1.274)$ & $4.08 \pm(1.412)$ & .523 \\
\hline $\begin{array}{l}\text { Post } 4 \text { Weeks } \\
\text { VAS }{ }^{b} \text { (in } \mathrm{cm} \text { ) }\end{array}$ & $1.17 \pm(1.239)$ & $1.50 \pm(1.383)$ & .384 \\
\hline
\end{tabular}

\section{DISCUSSION}

The study aimed to investigate the effect of gluteus maximus activation on subjects with anteriorly rotated sacroiliac joint dysfunction. Homogenous similarity recorded at baseline for gender, chronicity, and dominance.

ODI scores showed clinically significant change from $35 \%$ to $7.25 \%$ at four weeks following gluteus maximus activation exercises in experimental group, which is beyond the minimal clinically significant difference of 15\%.(16) A previous study on sacroiliac joint dysfunction showed a change in modified ODI from 38\% using MET, joint mobilization, ultrasound, and corrective exercises for four weeks. The reason for higher improvement could be due to additive effect of joint mobilization, ultrasound, MET, and corrective exercises. (15)

ODI scores showed clinically significant improvement from $37.7 \%$ to $14.5 \%$ at four weeks following flexion bias exercises in the control group. A literature stated that self-correction exercises, such as a knee to chest and sitting leaning forward, helps to prevent the recurrence of sacroiliac joint 
dysfunction, thus improving the functional status of the patient. (21) In the current study, the possible mechanism could be activation of abdominal muscles which maintained the sacroiliac integrity by restoring its self-bracing mechanism. (5)

ODI scores showed the disability index in experimental group of $7.25 \%$ compared to the control group which revealed $14.5 \%$ following four weeks of intervention which is clinically insignificant and thereby no difference in between groups. Studies have shown $15.5 \%$ as the minimal clinically significant difference for between-group analysis for ODI outcome measure.(16) The current study is in line with the previous study which showed no significant difference in ODI using joint mobilization and stabilization exercises following four weeks. ${ }^{(12)}$ On the contrary to the current study, a previous study showed a change in modified ODI by $10.42 \%$ using MET, joint mobilization, and corrective exercises. The possible reason for the difference could be activation of both abdominals and gluteus maximus. However, the current study targeted gluteus maximus activation alone.(22)

The immediate effect of MET on VAS scores showed a clinically significant change from $6.79 \mathrm{~cm}$ to $4.33 \mathrm{~cm}$ in gluteus maximus activation group and 6.79 $\mathrm{cm}$ to $4.08 \mathrm{~cm}$ in flexion bias exercises group. Studies have shown $2 \mathrm{~cm}$ as a minimal clinically significant change in the within-group analysis for VAS outcome measure. (15) The amount of improvement was seen following the previous study on sacroiliac joint dysfunction with a reduction in VAS scores by $2.20 \mathrm{~cm}$ using MET and joint mobilization. (23) Both the groups have improved from baseline to post-intervention and it is not statistically significant between groups.

In the present study, the reason for the reduction in VAS scores could be due to MET, in which small amplitude oscillatory and distraction movements stimulate the mechanoreceptors that inhibit the transmission of nociceptive stimuli at the spinal cord and brainstem levels. Isometric contraction to hip extensors was given, thus relieving the strain of the involved ligaments, relieving the tension of the nerve roots and anterior joint capsule of the sacroiliac joint. Correction of the mal-positioned pelvis reduces the pain by restoring the self-bracing position of sacroiliac joint and thus increases the range of motion of the joint during load transfer activities. 21,23$)$
Following four weeks of gluteus maximus activation, there was a reduction in VAS from $4.33 \mathrm{~cm}$ to $1.17 \mathrm{~cm}$ which is beyond clinically significant difference of $2 \mathrm{~cm}$. It is similar to the previous study by Mathew et al.(15) on sacroiliac joint dysfunction which showed improvement in VAS scores by $2.9 \mathrm{~cm}$ using corrective exercises which included activation of abdominals and gluteus maximus muscles for four weeks.

Following four weeks of flexion bias exercise intervention, there was a reduction in VAS scores from $4.08 \mathrm{~cm}$ to $1.50 \mathrm{~cm}$ which is beyond a clinically significant difference of $2 \mathrm{~cm}$. The study by Mathew et al. showed improvement in VAS scores by $2.93 \mathrm{~cm}$ using ultrasound, MET, and corrective exercises. The plausible reason for differences could be the additional use of ultrasound along with MET. ${ }^{(15)}$

VAS scores of between-group analysis demonstrated statistical improvement in experimental group $(1.17 \mathrm{~cm})$ compared to control group $(1.50 \mathrm{~cm})$. The minimal clinically significant change in VAS scores in between group is reported to be 2.5 points; hence, the change was not clinically significant. This finding is consistent with another study that found similar results using joint mobilization and stabilization exercises in patients with sacroiliac joint dysfunction. (12)

PALM scores of within-group analysis of experimental group showed significant improvement from $4.25^{\circ}$ to $2.00^{\circ}$ and in control group from $4.33^{\circ}$ to $2.00^{\circ}$ which is beyond the clinically significant change of $2^{\circ}-3^{\circ}$. To the best of our knowledge, the current study is first to use PALM device to measure pelvic angles for the subjects with anterior rotated sacroiliac joint dysfunction. The possible reason for pelvic angle correction could be because of isometric contraction given to hip extensors for five repetitions for about 5-10 sec hold.

The other mechanism for the change in pelvic tilt was because of MET which restores the self-bracing position of the pelvis by moving the PSIS posteriorly, caudally, and medially on the sacrum, which corrects the form closure of the sacroiliac joint thus achieving the neutral position of the pelvis. (20)

PALM scores were maintained at the end of four weeks to $2^{\circ}$ in both experimental as well as the control group. The possible reason for the improvement in both the groups could be due to the activation of the gluteus maximus and abdominals, respectively, 
forming the force closure of the pelvis, thus maintaining the self-bracing position during weight-bearing activities. ${ }^{(23)}$

\section{CONCLUSION}

A four-week gluteus maximus activation exercise and flexion bias exercise programme, along with MET technique, in subjects with anteriorly rotated sacroiliac joint dysfunction showed improvements in physical function which was assessed using Oswestry Disability Index. There was reduction in pain and normalization of pelvic tilt angle which was maintained at the end of the four-week programme in both groups.

Immediate improvements were seen in pain scores and pelvic tilt angles in both groups post-correction of anteriorly rotated sacroiliac joint dysfunction by MET.

This study should be viewed in the light of several limitations. The home programme was unsupervised for five days a week for four weeks. Assessor blinding for taking outcome measures was not done. The age group and chronicity of subjects varies in experimental and control groups.

Future research should be undertaken on the long-term effect of gluteus maximus activation exercises on subjects who have anteriorly rotated SIJD and investigate the incidence of relapse post-gluteus maximus activation exercises. The inclusion of EMG activity of gluteus maximus as an outcome measure on gluteus maximus activation for anteriorly rotated sacroiliac joint dysfunction can be used to investigate the proper firing of the muscle during weight-bearing activities.

\section{ACKNOWLEDGMENTS}

We thank all our subjects for their cooperation and participation in the study.

\section{CONFLICT OF INTEREST NOTIFICATION}

The authors declare there are no conflicts of interest.

\section{COPYRIGHT}

Published under the CreativeCommons Attribution-NonCommercial-NoDerivs 3.0 License.

\section{REFERENCES}

1. Smidt G, Wei SH, McQuade K, Barakatt E, Sun T, Stanford W. Sacroiliac motion for extreme hip positions: a fresh cadaver study. Spine J. 1997;22(18):2073-2082.

2. Hansen H, Manchikanti L, Simopoulos TT, Christo PJ, Gupta S, Smith HS, et al. A systemic evaluation of the therapeutic effectiveness of sacroiliac joint interventions. Pain Physician. 2012;15(3):247-278.

3. Punjabi M. Lumbar spine instability: a biomechanical challenge. Curr Orthop Pract. 1994;8(2):100-105.

4. Richardson C, Jull G, Hodges P, Hidus J. Therapeutic Exercise for Spinal Segment Stabilization in Low Back Pain. Scientific Basis and Clinical Approach, 1st ed. Edinburgh: Churchill Livingstone; 1999.

5. Vleeming A, Schuenke MD, Masi AT, Carreiro JE, Danneels L, Willard FH. The sacroiliac joint: an overview of its anatomy, function and potential clinical implications. J Anat. 2012;221(6):537-567.

6. van Wingerden JP, Vleeming A, Buyruk HM, Riassadak K. Stabilization of the sacroiliac joint in vivo: verification of muscular contribution of force closure of the pelvis. Eur Spine J. 2004;13(3):199-205.

7. Leibenson $\mathrm{C}$. The relationship of the sacroiliac joint, stabilization musculature and lumbo-pelvic instability. J Bodyw Mov Ther. 2004;8(1):42-45.

8. Lee D. The Pelvic Girdle-an Approach to Examine and Treatment of the Lumbo-Pelvic-Hip Region, 3rd ed. Toronto, ON: Elsevier Canada; 2004.

9. DonTigny RL. Evaluation, manipulation and management of anterior dysfunction of the sacroiliac. The DO (a publication of the Am. Osteopathic Assoc.). 1973;14(1):215-226.

10. Hossain M, Nokes LDM. A model of dynamic sacroiliac joint instability from malrecruitment of gluteus maximus and biceps femoris muscles resulting in low back pain. Med Hypotheses. 2005;65(2):278-281.

11. Lasett M. Evidence-based diagnosis and treatment of the sacroiliac joint. J Man and Manip Ther. 2008;16(3):142-152.

12. Fahimeh K, Mehdi Z, Ali G, Abbas A, Soha B. Comparison of manipulation and stabilization exercises in patients with sacroiliac joint dysfunction patients: a randomized clinical trial. J Bodyw Mov Ther. 2019;23(1):177-182.

13. Marco AN, Diego GF, Karina TK, Robroy LM, Thiago YF. Strengthening the gluteus maximus in subjects with sacroiliac joint dysfunction. Int J Sports Phys Ther. 2018;13(1):114.

14. Hungerford B, Gilleard W, Hodges P. Evidence of altered lumbopelvic muscle recruitment in the presence of sacroiliac joint pain. Spine $J$. 2003;28(14):1593-1600.

15. Mathew R, Srivastava N, Joshi S. A study to compare the effectiveness of MET and joint mobilization along with conventional physiotherapy in the management of SI joint dysfunction in young adults. Indian J Physiother Occup Ther. 2015;9:203-208. 
16. Copay AG, Cher DJ. Is the Oswestry Disability Index a valid measure to respond to sacroiliac joint treatment? Qual Life Res. 2016;25(2):283-292.

17. Fairbank JC, Pynsent PB. The Oswestry Disability Index. Spine J. 2000;25(22): 2940-2953.

18. Hagins M, Brown M, Cook C, Gstalder K, Kam M, Kominer $\mathrm{G}$, et al. Intratester and intertester reliability of the Palpation Meter (PALM) in measuring pelvic position. J Man Manip Ther. 1998;6(3):130-136.

19. Petrone M, Guinn J, Reddin A, Sutlive T, Flynn T, Garber M. The accuracy of the palpation meter (PALM) for measuring pelvic crest height difference and leg length discrepancy. J Orthop Sports Phys Ther. 2003;33(6):319-325.

20. Boonstra AM, Preuper HR, Reneman MF, Posthumus JB, Stewart RE. Reliability and validity of the visual analogue scale for disability in patients with chronic musculoskeletal pain. Int J Rehabil Res. 2008;31(2):165-169.
21. Cailliet R. Low Back Pain Syndrome, 2nd ed. Philadelphia, PA: FA Davis Co.; 1968.

22. Bindra S. A study on the efficacy of muscle energy technique as compared to conventional therapy on lumbar spine range of motion on chronic low back pain of sacroiliac origin. Human Biol Rev. 2013;2(4):13.

23. Don Tingy RL. Critical analysis of the sequence and extent of the result of the pathological failure of the self- bracing of the sacroiliac joint. J Man and Manip Ther. 1999;7(4):173-181.

Corresponding authors: Venkatesan Prem, PT, PHD, Department of Physiotherapy, Manipal University, School of Allied Health Sciences, Manipal College of Health Professions, Bangalore, India-560064.

E-mail: prem.v@manipal.edu 